\title{
Large area assembly of patterned nanoparticles by a polydimethylsiloxane template
}

\author{
Xiangdong $\mathrm{Ye}^{1^{*}}$, Anjiang $\mathrm{Cai}^{1}$, Jinyou Shao ${ }^{2}$, Xiaojun $\mathrm{Wu}^{1}$, Xiaoguang Ruan ${ }^{1}$ and Xuefeng Zhang ${ }^{1}$
}

\begin{abstract}
A technique in which a polydimethylsiloxane (PDMS) template is used to assemble silicon oxide nanoparticles is proposed herein. The PDMS substrate was first constructed in a series of casting steps and a copper mask was then made with a laser marking system. Silicon oxide nanoparticles were generated by a modified Stober method. A PDMS substrate covered by the copper mask was then coated with a metal Au layer via sputtering to form the PDMS template. Finally, the silicon oxide nanoparticles were spin-coated on the PDMS template. Because the PDMS template consists of highly hydrophobic PDMS and less hydrophilic Au patterns, the nanoparticles undergo a dual dewetting process on the PDMS template to form an annular distribution around the borders of the Au patterns.
\end{abstract}

\section{INTRODUCTION}

The assembly of ordered patterns of nanoparticles on solid substrates has attracted much attention due to the possible applications of such assemblies in various areas, such as flexible electronics [1,2], biological sensors [3], photonic crystals [4], photoelectrical devices, and data storage $[5,6]$, etc.

Compared with rigid substrates that can utilize traditional techniques, the assembly of nanoparticles on flexible substrates is unusual. Many techniques have been developed for patterning nanoparticles on flexible substrates. Seung et al. [7] combined inkjet printing and selective laser sintering to assemble metal nanoparticles on a polymer substrate. Inkyu et al. [8] adopted nano-imprinting to directly pattern a metallic nanoparticle solution on a flexible substrate. He et al. [9] proposed a simple but rapid approach for synthesis of single-crystalline $\mathrm{Co}_{3} \mathrm{O}_{4}$ nanorods on silicon substrates, which is compatible with microtechnology and can be used to fabricate metal oxide nanorods at low substrate temperatures with large-scale applicability. Lee et al. [10] obtained a smooth $\mathrm{SiO}_{x}$ thin film by atmospheric pressure plasma-enhanced chemical vapor deposition based on the controlled formation of nanopar- ticles to decrease the surface roughness of the film by optimizing the flow rate of PDMS, oxygen, and helium. Sun et al. [11] developed a novel patterning technique to create three-dimensional (3D) patterns of micro- and nanoparticle assemblies by utilizing evaporative self-assembly based on the coffee-ring effect of an evaporating suspension. Researchers in Israel [12] reported a simple method for patterning nanoparticles by combining self-assembly with sintering of silver nanoparticles at room temperature. Yeo et al. [13] indicated that direct metal patterning based on laser-induced local melting of metal nanoparticle ink is a promising low-temperature alternative to conventional metal patterning processes. Metal nanoparticles can also be produced by the electrodeposition process. For example, Liu et al. [14] prepared platinum (Pt) particles on an indium tin oxide (ITO) substrate by pulsed electrodeposition and investigated the electrocatalytic properties of these species in methanol oxidation. Moreover, hierarchical platinum particles can be synthesized by the electro-deposition technique. Zhong and coworkers [15] prepared hierarchical Pt nanoparticles with various morphologies, including sheet-, flower-, prickly sphere-, and cauliflower-like shapes by controlling the electrodeposition parameters. Du et al. [16] proposed a surfactant-free and template-free electrochemical approach for generating well-dispersed $\mathrm{Pt}$ nanosheets. By simply changing the anions from $\mathrm{SO}_{4}{ }^{2-}$ to $\mathrm{Cl}^{-}$in the electrodeposition solution, the generated $\mathrm{Pt}$ deposits changed from flower-like Pt particles consisting of aggregated Pt nanosheets to well-dispersed Pt nanosheets.

Lee et al. [17] assembled Ag nanoparticles into well-ordered line arrays via anisotropic buckling templates with a subsequent transfer process, where a small amount of water rather than glue materials was employed. Chang et al. [18] proposed a novel technique for fabricating copper patterns by a patterning-adsorption-plating process, in which ion-adsorption nanoparticles were inkjet printed

\footnotetext{
${ }^{1}$ School of Mechanical and Electrical Engineering, Xi'an University of Architecture and Technology, Xi'an 710055, China

${ }^{2}$ State Key Laboratory of Manufacturing Systems Engineering, Xi'an Jiaotong University, Xi'an 710049, China

*Corresponding author (email: yexiangd@xauat.edu.cn)
} 
on a polyethylene terephthalate (PET) substrate and the metal patterns were subsequently produced by electroless plating. Cyrille et al. [19] devised a reliable technique to obtain nanoparticle supercrystal arrays by confining a gold nanoparticle colloid between a patterned PDMS mold and a flat substrate. Roller et al. [20] presented the fabrication of self-assembled ring-shaped plasmonic metamolecules by utilizing DNA origami structures as templates for the synthesis of a wide variety of ring geometries containing nanoparticles.

In this paper, we propose a simple technique for fabricating micro-patterns of silicon oxide $\left(\mathrm{SiO}_{2}\right)$ nanoparticles on a flexible substrate. The detailed process includes the fabrication of a PDMS template and a spin-coating step. Firstly, a copper film is patterned by a laser marking system to obtain a copper mask with round hole and square hole arrays. Subsequently, the copper mask is placed on the surface of the PDMS substrate, and the assembly is placed into a sputter to obtain the metal coating. Under the mask of the patterned copper film, the metal patterns (such as the round patterns and the square patterns) emerge on the PDMS substrate. The PDMS template is thus generated for assembly of the $\mathrm{SiO}_{2}$ nanoparticles. Silicon oxide nanoparticles are generated by the modified Stober Method [21]. Finally, the obtained solution with the $\mathrm{SiO}_{2}$ nanoparticles is spin-coated on the PDMS template. The $\mathrm{SiO}_{2}$ nanoparticles wet the metal $\mathrm{Au}$ areas and dewet the PDMS areas, leading to their assembly on the PDMS template. The advantages of using the PDMS template to assemble the silicon oxide nanoparticles include the simplicity and low cost of the process and its convenience for large area assembly.

\section{EXPERIMENTAL SECTION}

\section{Fabrication of the PDMS template with the patterned $\mathrm{Au}$} After thoroughly mixing PDMS and the curing agent (ESSIL 291 and ESSIL 293, Axson Corp.) in a ratio of 10:1 by weight, the mixture was degassed and cast on the polished surface of a silicon wafer. The assembly was then cured in an oven at $80^{\circ} \mathrm{C}$ or $3 \mathrm{~h}$. The PDMS substrate was obtained by peeling the PDMS from the silicon wafer.

A copper film, used as the mask material, was patterned by a laser marking system (Han's Laser Technology Co., Ltd.), where the obtained copper mask contained the round hole and square hole arrays.

The obtained copper mask was placed on the surface of the PDMS substrate and the assembly was placed into an ion sputter (E-1045, Hitachi Japan) to obtain a metal Au coating. The sputtering current was $35 \mathrm{~mA}$ and the time was $70 \mathrm{~s}$. After peeling the copper mask from the PDMS substrate, the PDMS template with the patterned Au was obtained.

\section{Preparation of $\mathrm{SiO}_{2}$ nanoparticles by the modified Stober Method}

Firstly, $4 \mathrm{~mL}$ of ammonium hydroxide was added to $50 \mathrm{~mL}$ of anhydrous ethanol and stirred for $30 \mathrm{~min}$. Then, $1.1 \mathrm{~mL}$ of tetraethylorthosilicate (TEOS) was added to the above solution, forming a $\mathrm{SiO}_{2}$ nanoparticle suspension after stirring for $4 \mathrm{~h}$. An aliquot of the suspension $(11 \mathrm{~mL})$ was withdrawn and ultrasonically dispersed for $10 \mathrm{~min}$, and the $\mathrm{SiO}_{2}$ nanoparticles were centrifugally separated from the solvent. The supernatant was removed, and the precipitate was dispersed in deionized water. Finally, the $\mathrm{SiO}_{2}$ nanoparticles were separated from the deionized water by centrifugation and dispersed in $11 \mathrm{~mL}$ of anhydrous ethanol.

\section{Assembly of the $\mathrm{SiO}_{2}$ nanoparticles on the PDMS template}

The obtained solution of the $\mathrm{SiO}_{2}$ nanoparticles was spin-coated on the PDMS template at $1000 \mathrm{rpm}$ for $40 \mathrm{~s}$. Because the PDMS template consists of high surface energy areas (the metal Au areas) and low surface energy areas (the PDMS areas), the $\mathrm{SiO}_{2}$ nanoparticles wet the metal $\mathrm{Au}$ areas but dewet the PDMS areas. The patterned $\mathrm{SiO}_{2}$ nanoparticles on the PDMS template were thus obtained.

\section{RESULTS AND DISCUSSION}

The thickness of the copper film is $30 \mu \mathrm{m}$, and the film could be patterned by the laser marking system using a current of 20-24 A. The obtained copper mask with the arrays of round holes and square holes is shown in Fig. 1a. After coating the PDMS substrate (covered by the copper mask) with the metallic Au by ion sputtering, the PDMS template was generated as shown in Fig. $1 \mathrm{~b}$.

The PDMS template contains two kinds of Au patterns: the round dot array and the square dot array, as shown in Fig. 2. It is obvious that the fidelity of the patterned round dots or square dots is not very high because the fidelity of the copper mask obtained by the laser marking system is limited. From Figs $2 c$ and d, the width of the square dots and the diameter of the round dots were both determined to be about $170 \mu \mathrm{m}$.

In order to determine the thickness of the patterned $\mathrm{Au}$ dots on the PDMS template, a polished silicon substrate was coated with $\mathrm{Au}$ by sputtering under the aforementioned conditions (current: $35 \mathrm{~mA}$; time: $70 \mathrm{~s}$ ). After checking the cross-section of the silicon substrate by scanning electron microscopy (SEM), it was deduced that the 
a

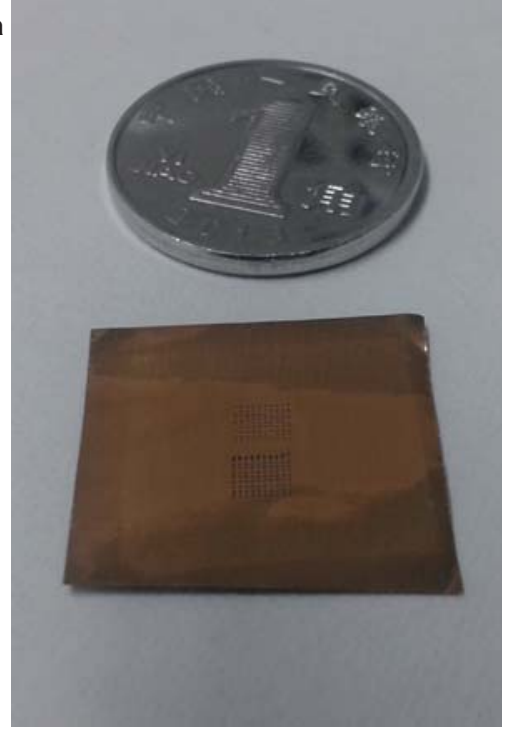

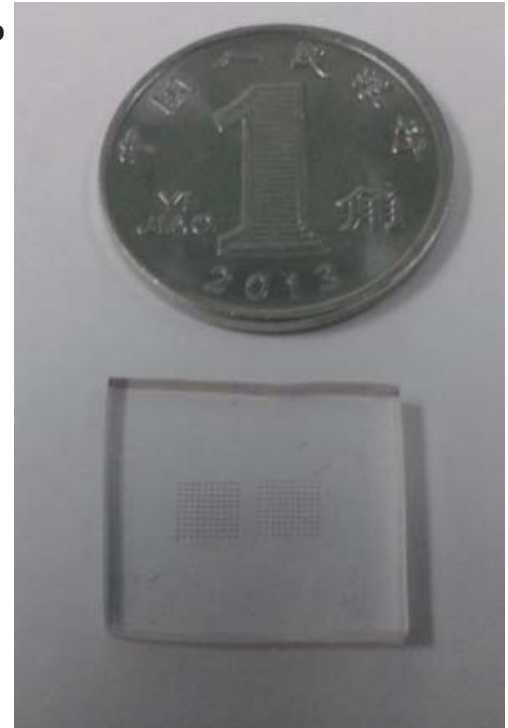

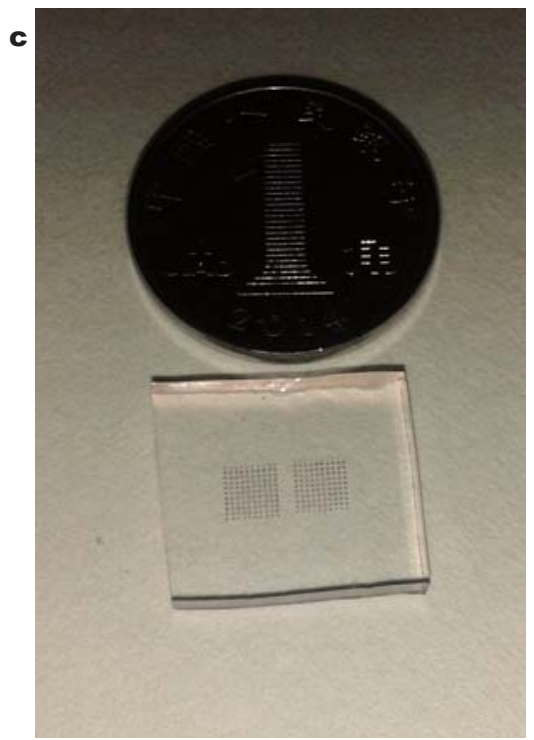

Figure 1 Photos of the patterned copper mask (a), the PDMS template (b), and PDMS coated with the patterned $\mathrm{SiO}_{2}$ nanoparticles (c).

$\mathbf{a}$
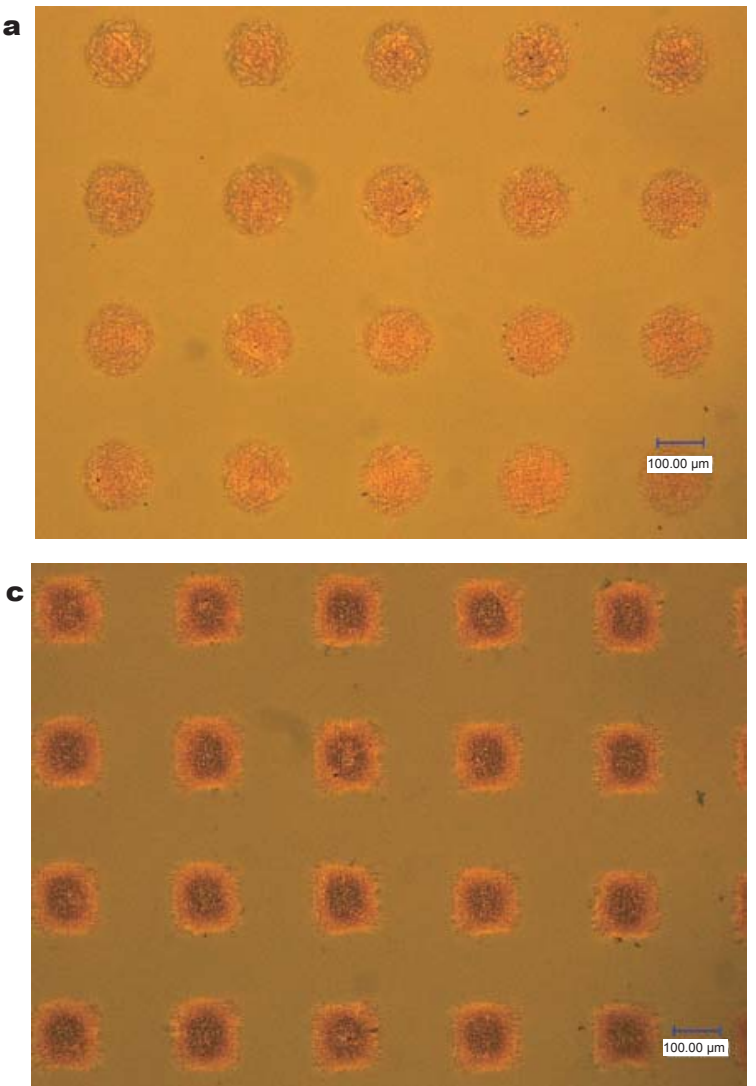

b

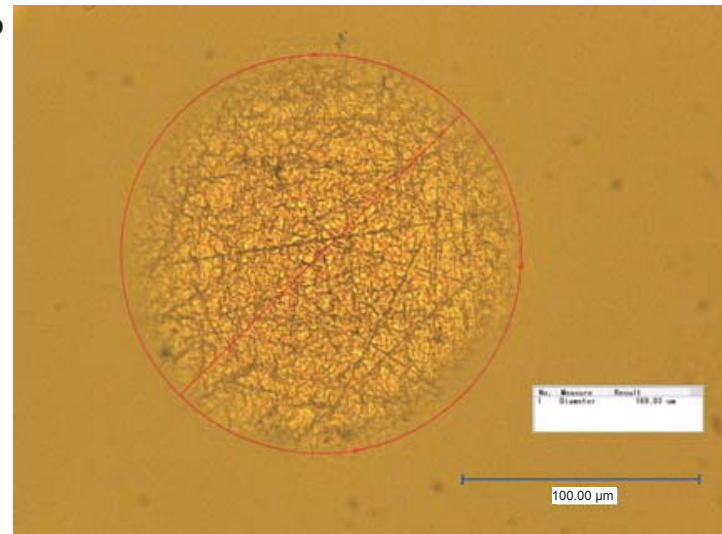

d

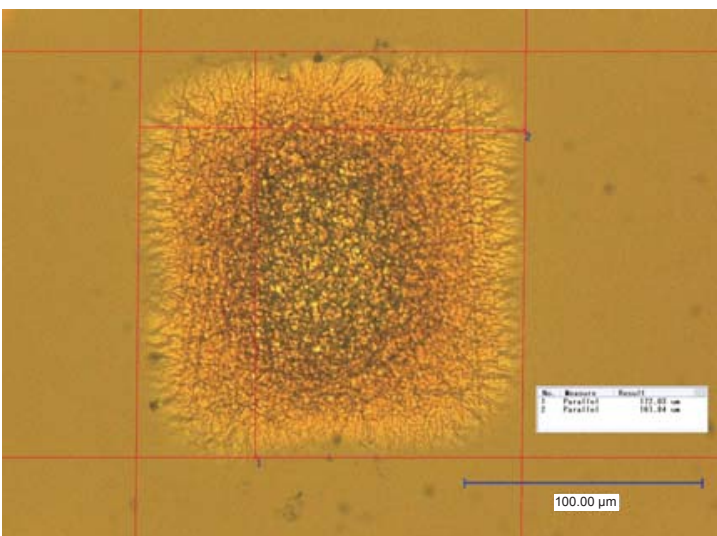

Figure 2 Optical microscopic images of PDMS template with the Au pattern: (a) the round dot array, (b) a single round dot, (c) the square dot array, (d) a single square dot. 
thickness of the Au patterns on the PDMS template was approximately $20 \mathrm{~nm}$.

We used the spin-coating process to assemble the $\mathrm{SiO}_{2}$ nanoparticles on the PDMS substrate. An ethanol solution of the $\mathrm{SiO}_{2}$ nanoparticles was spin-coated on the PDMS template at $1000 \mathrm{rpm}$ for $40 \mathrm{~s}$. We obtained PDMS coated with the patterned $\mathrm{SiO}_{2}$ nanoparticles, as shown in Fig. 1c.

The patterned PDMS template was inspected by optical microscopy (Fig. 3). Comparison of the images in Fig. 3 with those in Fig. 2 clearly indicates that the boundaries of the $\mathrm{Au}$ dots (round dots and square dots) are covered by the $\mathrm{SiO}_{2}$ nanoparticles and these species are mainly annular. Moreover, from Fig. 3, it is apparent that the inner sides of these species remains on the borders of the Au dots and the outer borders of these species extended beyond those of the Au dots.

In order to verify whether the adhering species were $\mathrm{SiO}_{2}$ nanoparticles, the coated PDMS template was subjected to energy dispersive spectroscopy (EDS) and SEM. These results are shown in Figs 4, 5 and 6, respectively.

From Fig. 4, it is obvious that the adhering species are
$\mathrm{SiO}_{2}$ nanoparticles as anticipated. Figs 5 and 6 show the SEM images of the round dot and the square dot on the coated PDMS template.

From Figs 5 and 6, it is interesting that the distribution of the nanoparticles is uneven. Most of the nanoparticles are spread annularly along the outlines of the round Au dots or the square $\mathrm{Au}$ dots, whereas in the extensive central areas of the Au dots, there are only a few or even no nanoparticles. For example, in Figs 5a and e, a few nanoparticles are seen to be distributed at the top area of the circular Au dot. However, as shown in Fig. 6a, the entire area of the square Au dot is bare except for the boundary areas.

To elucidate the reason for the strange distribution of the nanoparticles, we checked the entire bare area of the Au dots and found that the surface of the Au dots consisted of many wrinkles and the width of the wrinkles was approximately $50 \mathrm{~nm}$, as shown in Figs $5 \mathrm{f}$ and 6d. In comparison, the same PDMS substrate (without the copper mask) was sputtered with Au metal under the same conditions (sputtering current: $35 \mathrm{~mA}$; time: $70 \mathrm{~s}$ ). The obtained Au coating on the PDMS substrate was evaluated by SEM (Fig. 7).
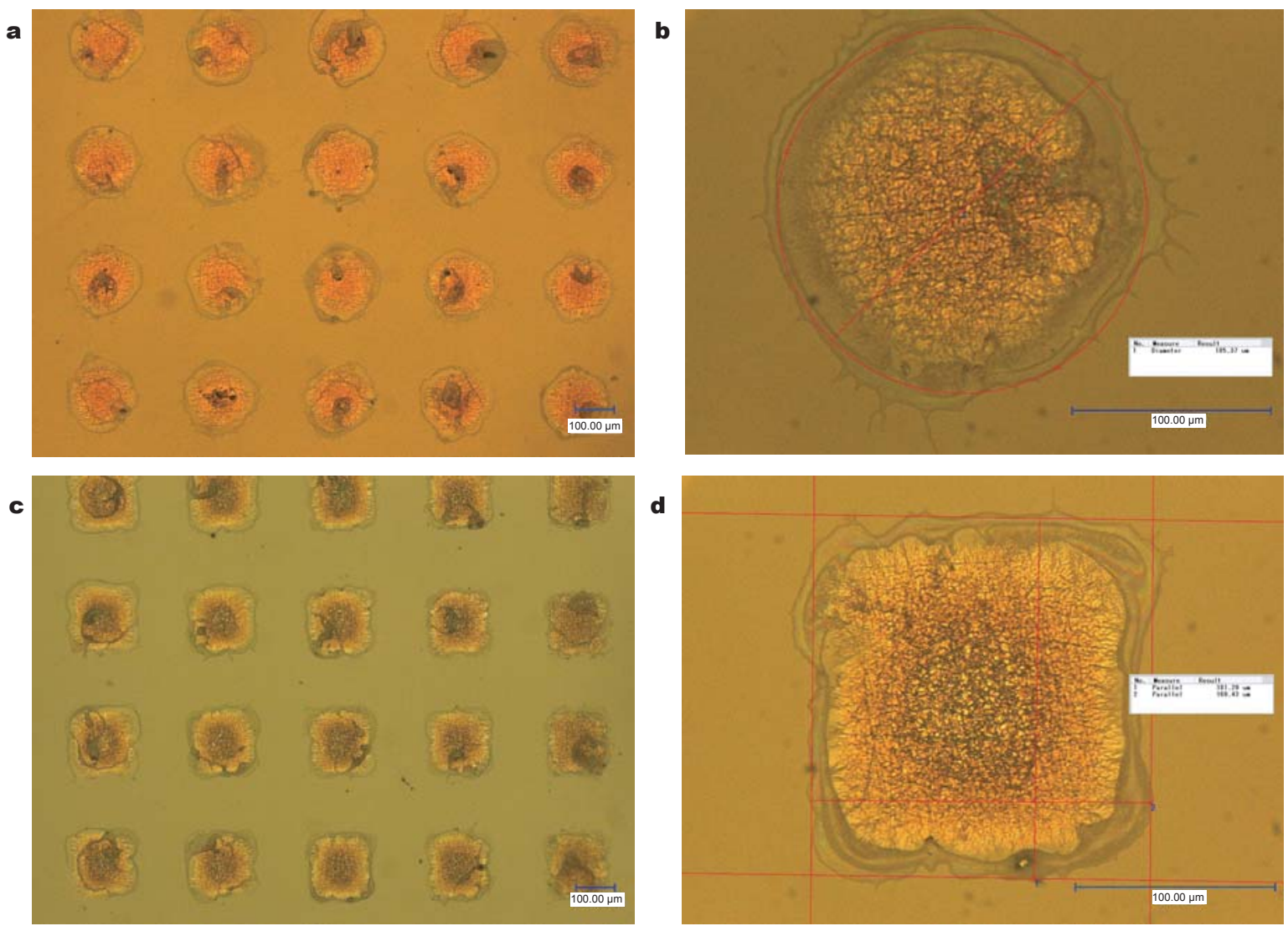

Figure 3 Optical microscopic images of PDMS template coated with $\mathrm{SiO}_{2}$ nanoparticles: (a) the round dot array, (b) a single round dot, (c) the square dot array, (d) a single square dot. 

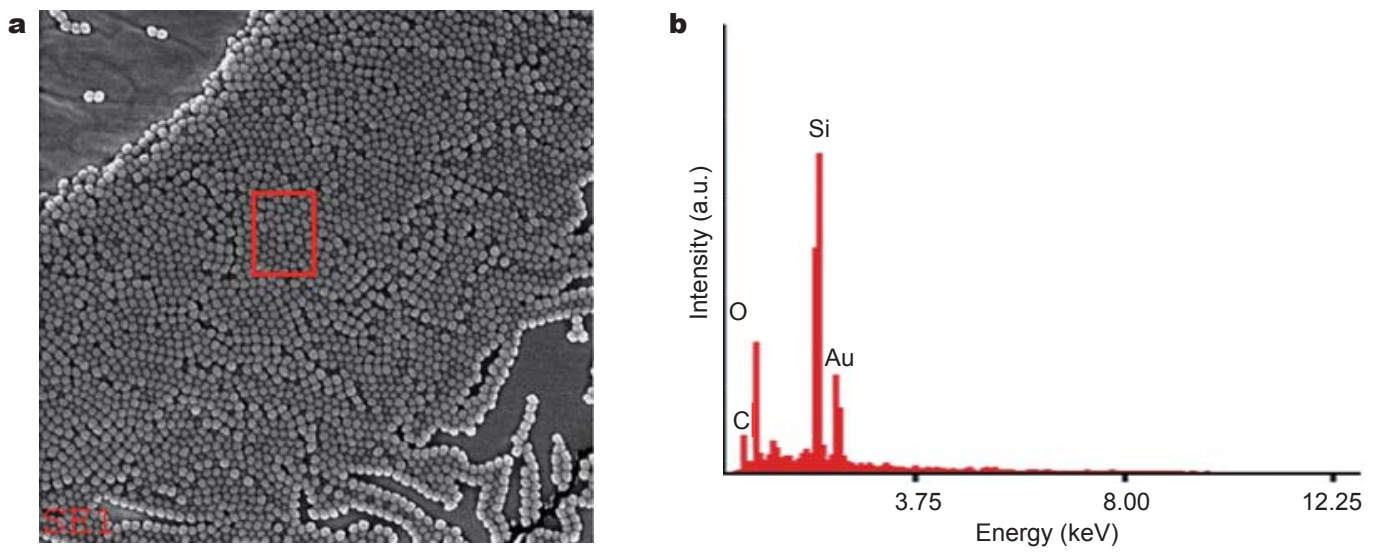

Figure 4 (a) SEM image of the coated PDMS template (the red square denotes the areas inspected by EDS), (b) EDS results for the areas enclosed by red squares.
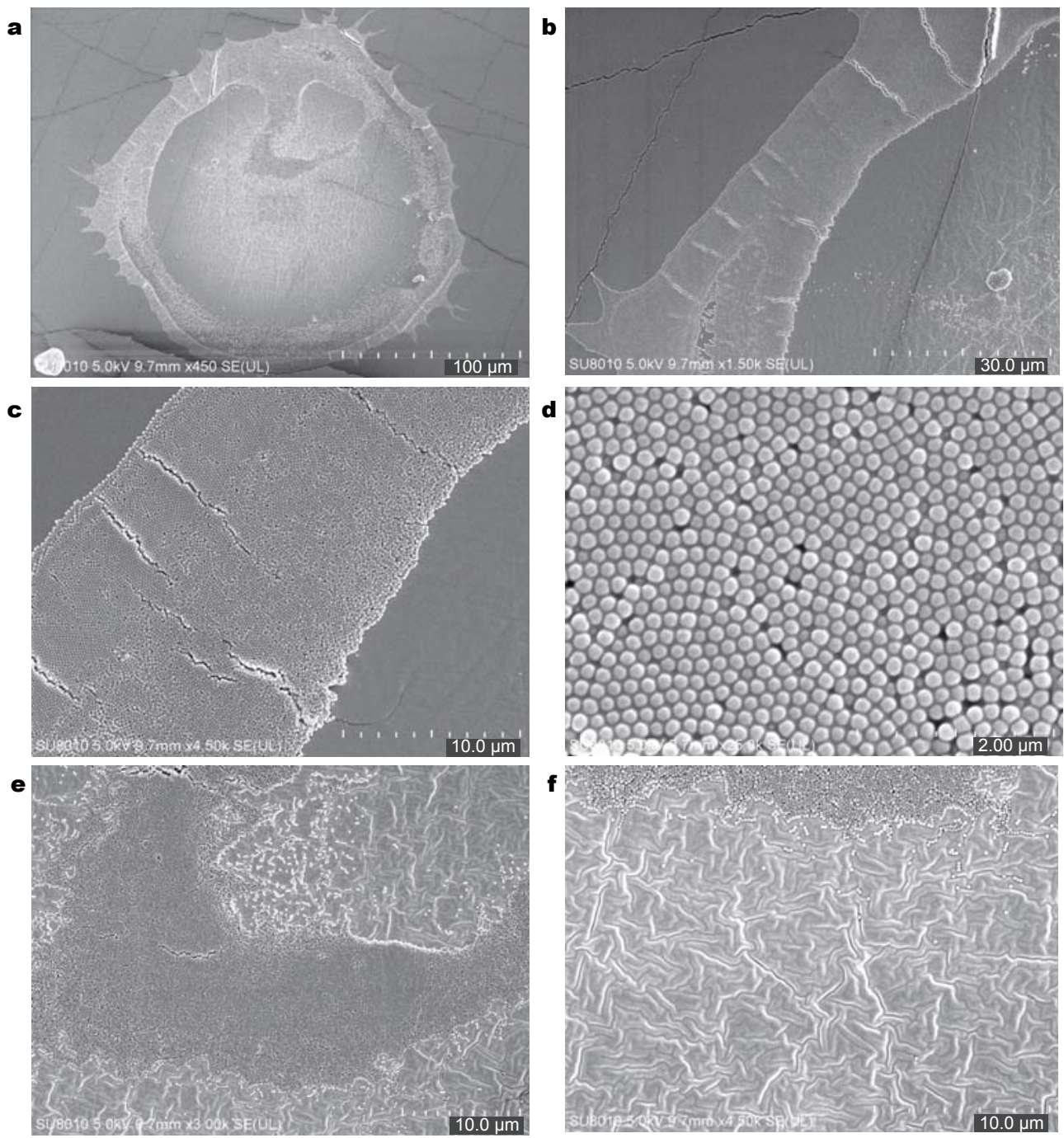

Figure 5 SEM images of a single round dot (the same dot in Fig. 3b) on the PDMS template coated with $\mathrm{SiO}_{2}$ nanoparticles: (a) overall image of the round dot, (b) boundary of the round dot, (c and d) magnified image of the boundary, (e) the near-center area covered with the nanoparticles, (f) bare $\mathrm{Au}$ area without the nanoparticles. 
a

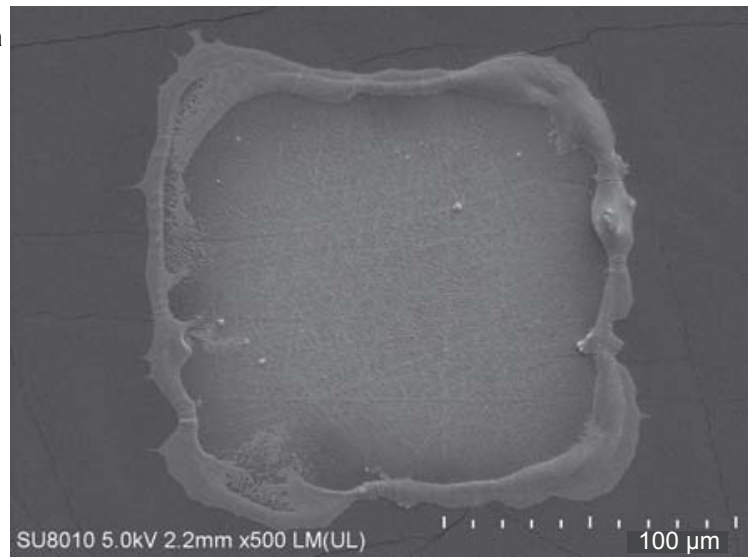

c

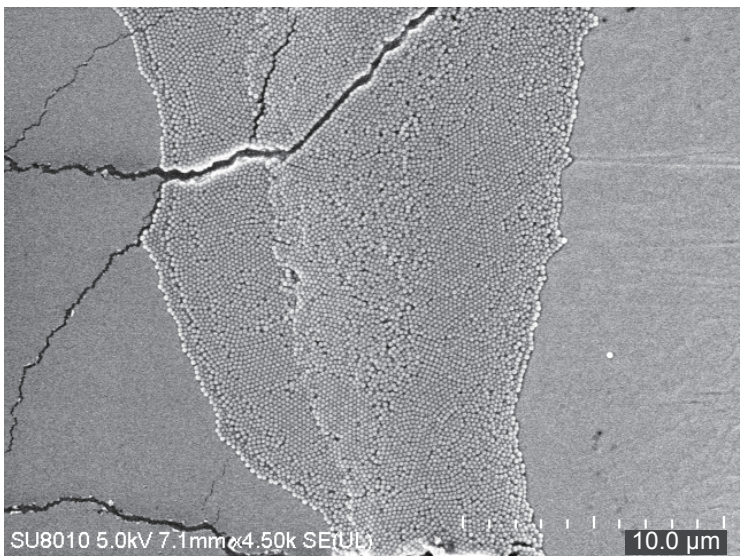

b

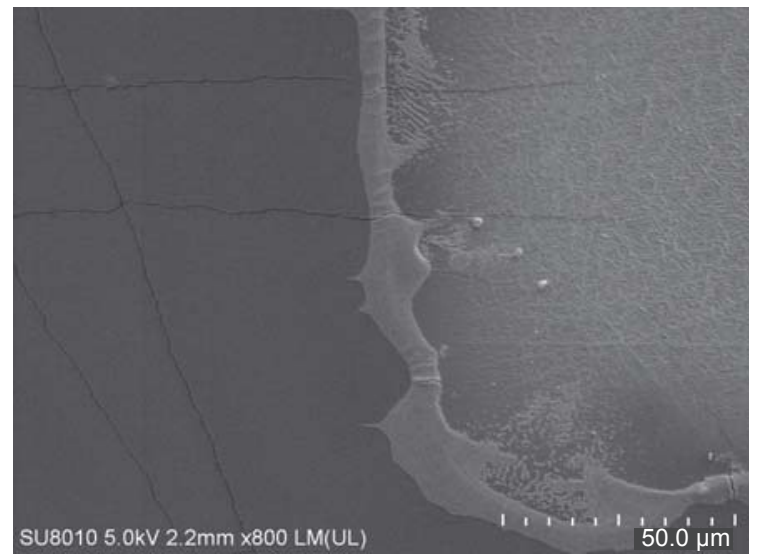

d

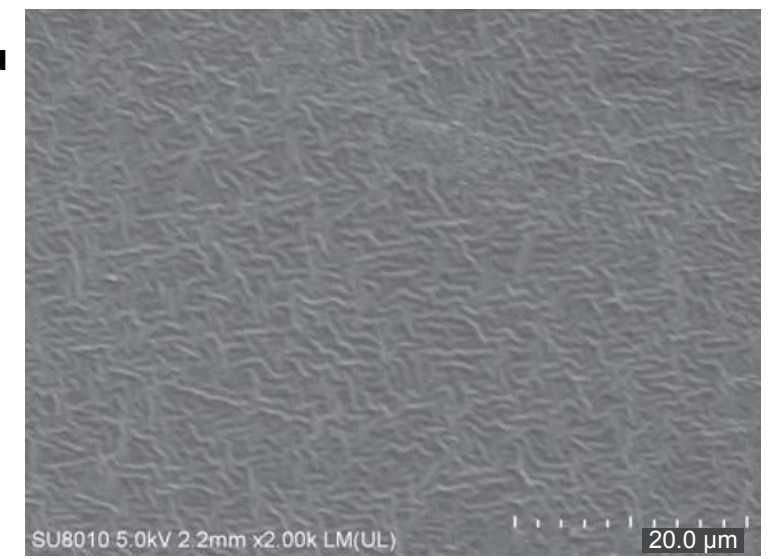

Figure 6 SEM images of a single square dot (the same dot in Fig. 3d) on the PDMS template coated with $\mathrm{SiO}_{2}$ nanoparticles: (a) overall image of the square dot, (b) boundary of the square dot, (c) magnified image of the boundary, (d) bare Au area without the nanoparticles.

Fig. 7 shows that the Au coating on the PDMS is not smooth but full of wrinkles. The wrinkles may affect the wettability of the Au coating. Thus, we measured the contact angle of water drops on the Au coating (Fig. 8).

The contact angle of water on the bare PDMS substrate is as high as $112^{\circ}$ (Fig. 8a), which verifies the high hydrophobicity of the PDMS surface. Fig. $8 \mathrm{~b}$ shows that the contact angle of water on the PDMS substrate coated with the $\mathrm{Au}$ layer is approximately $55^{\circ}$, indicating that the wrinkled $\mathrm{Au}$ layer is hydrophilic. When the wrinkles in the Au layer were removed by deposition of Au metal on a polished silicon substrate, the contact angle was reduced to $37^{\circ}$ (Fig. 8c). The data indicate that the smooth Au layer is much more hydrophilic than the wrinkled Au layer. Thus, we propose that the annular distribution of the nanoparticles on the PDMS template can be explained as presented in Fig. 9.

When the ethanol solution containing the nanoparticles is spin-coated on the PDMS template, the solution undergoes volatilization and the nanoparticles will be left on the

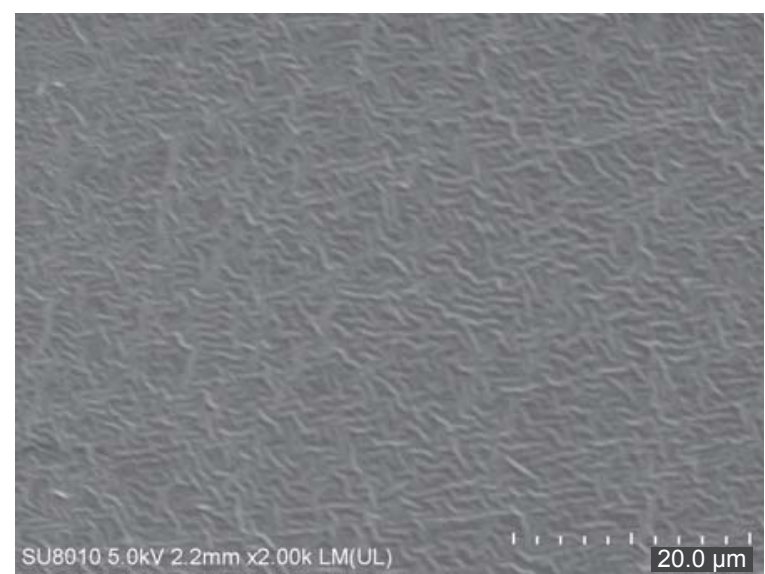

Figure 7 SEM image of the Au coating on the PDMS substrate without the copper mask.

template. Because the PDMS template includes the PDMS areas (the hydrophobic areas) and the Au pattern areas (the hydrophilic areas), the nanoparticles will dewet on the PDMS areas and wet on the Au pattern areas. We propose 
a

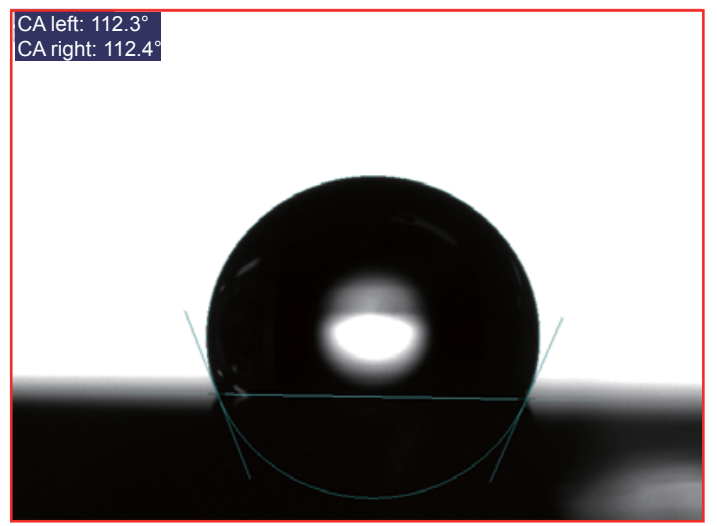

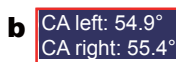

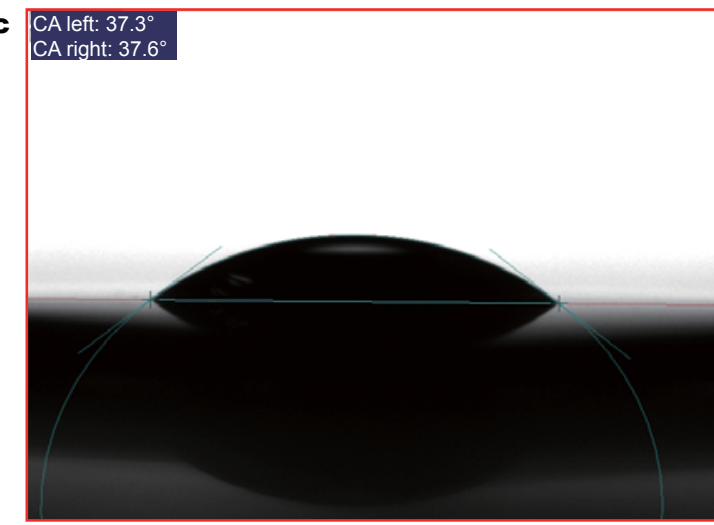

Figure 8 Contact angles of water drops on the surface of (a) bare PDMS substrate, (b) PDMS substrate coated with Au layer, (c) silicon substrate coated with Au layer.

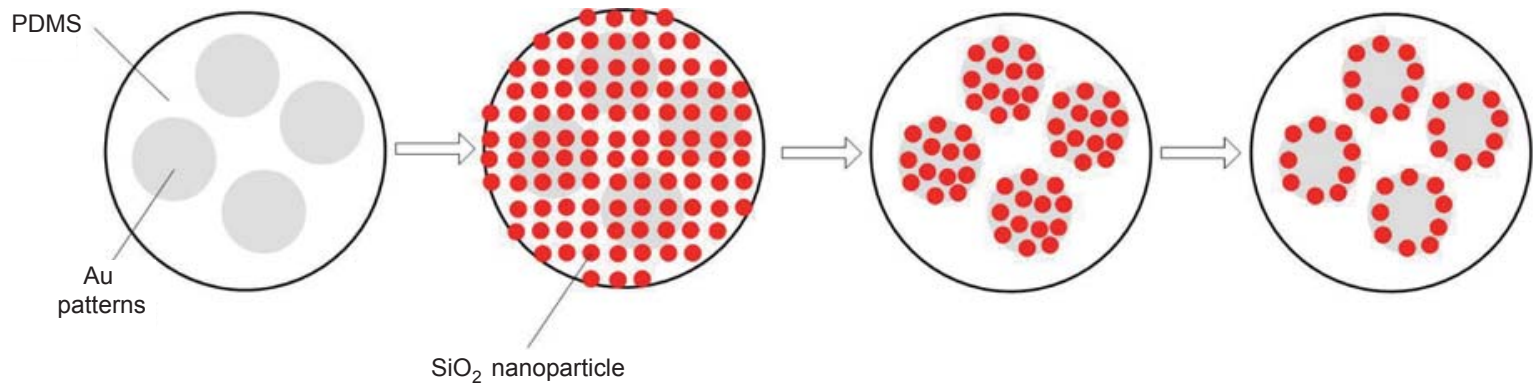

Figure 9 Schematic representation of the two-step dewetting process for $\mathrm{SiO}_{2}$ nanoparticles on the PDMS template.

that this step is the first dewetting process of the nanoparticles. However, because the wrinkles in the Au pattern decrease the hydrophilicity of the surface, the nanoparticles will dewet again on the Au pattern areas. We propose that this is the second dewetting process of the nanoparticles. After the second dewetting, the nanoparticles in the central areas of the Au pattern will move to the borders of the Au pattern with consequent emergence of annular structures of the nanoparticles around the Au patterns.

\section{CONCLUSIONS}

In conclusion, through a simple spin-coating process, $\mathrm{SiO}_{2}$ nanoparticles were assembled on a PDMS template comprising Au patterns on the PDMS substrate. The assembled nanoparticles on the PDMS substrate are distributed annularly around the borders of the Au patterns. We propose that the annular distribution of the nanoparticles results from dual dewetting of the nanoparticles on the PDMS template due to the high hydrophobicity of PDMS and the 
decreased hydrophilicity of the Au patterns.

Received 1 August 2015; accepted 28 September 2015; published online 6 November 2015

1 Ahn BY, Duoss EB, Motala MJ, et al. Omnidirectional printing of flexible, stretchable, and spanning silver microelectrodes. Science, 2009, 323: 1590-1593

2 Briseno AL, Mannsfeld SCB, Ling MM, et al. Patterning organic single-crystal transistor arrays. Nature, 2006, 444: 913-917

3 Lee SH, Cho B, Yoon S, et al. Printing of sub-100-nm metal nanodot arrays by carbon nanopost stamps. ACS Nano, 2011, 5: 5543-5551

4 Norris DJ. Photonic crystals: a view of the future. Nat Mater, 2007, 6: $177-178$

5 Su B, Wang ST, Ma J, et al. "Clinging-microdroplet" patterning upon high-adhesion, pillar-structured silicon substrates. Adv Funct Mater, 2011, 21: 3297-3307

6 Robinson AP, Burnell G, Hu MZ, et al. Controlled, perfect ordering in ultrathin anodic aluminum oxide templates on silicon. Appl Phys Lett, 2007, 91: 143123

7 Seung HK, Heng P, Costas PG, et al. All-inkjet-printed flexible electronics fabrication on a polymer substrate by low-temperature high-resolution selective laser sintering of metal nanoparticles. Nanotechnology, 2007, 18: 345202

8 Inkyu $\mathrm{P}$, Seung HK, Heng $\mathrm{P}$, et al. Nanoscale patterning and electronics on flexible substrate by direct nanoimprinting of metallic nanoparticles. Adv Mater, 2008, 20: 489-496

9 He L, Li Z, Zhang Z. Rapid, low-temperature synthesis of singlecrystalline $\mathrm{Co}_{3} \mathrm{O}_{4}$ nanorods on silicon substrates on a large scale. Nanotechnology, 2008, 19: 155606

10 Lee JH, KimYS, Oh JS, et al. Characteristics of $\mathrm{SiO}_{x}$ thin film deposited by atmospheric pressure plasma-enhanced chemical vapor deposition using $\mathrm{PDMS} / \mathrm{O}_{2} / \mathrm{He}$. J Electrochem Soc, 2009, 156: $248-252$

11 Sun C, Stefano S, Albert PP, et al. Coffee-ring effect-based three dimensional patterning of micro/nanoparticle assembly with a single droplet. Langmuir, 2010, 26: 11690-11698

12 Michael L, Shlomo M. Flexible transparent conductive coatings by combining self-assembly with sintering of silver nanoparticles performed at room temperature. J Mater Chem, 2011, 21: 15378-15382
13 Yeo J, Hong S, Lee D, et al. Next generation non-vacuun, maskless, low temperature nanoparticle ink laser digital metal patterning for a large area flexible electronics. Plos One, 2012, 7: e42315

14 Liu J, Zhong C, Du X, et al. Pulsed electrodeposition of Pt particles on indium tin oxide substrates and their electrocatalytic properties for methanol oxidation. Electrochimica Acta, 2013, 100: 164-170

15 Liu J, Hu W, Zhong C, et al. Surfactant-free electrochemical synthesis of hierarchical platinum particle electrocatalysts for oxidation of ammonia. J Power Sources, 2013, 223: 165-174

16 Du XT, Yang Y, Liu J, et al. Surfactant-free and template-free electrochemical approach to prepare well-dispersed $\mathrm{Pt}$ nanosheets and their high electrocatalytic activities for ammonia oxidation. Electrochimica Acta, 2013, 111: 562-566

17 Lee J, Seo J, Kim D, et al. Capillary force-induced glue-free printing of Ag nanoparticle arrays for highly sensitive SERS substrates. ACS Appl Mater, 2014, 6: 9053-9060

18 Chang Y, Yang C, Zheng X, et al. Fabrication of copper patterns on flexible substrate by patterning-adsorption-plating process. ACS Appl Mater Interfaces, 2014, 6: 768-772

19 Cyrille H, Sergey N, Leonardo S, et al. Hierarchical self-assembled of gold nanoparticles into patterned plasmonic nanostructures. ACS Nano, 2014, 8: 10694-10703

20 Roller E, Khorashad LK, Fedoruk M, et al. DNA-assembled nanoparticle rings exhibit electric and magnetic resonances at visible frequencies. Nano Lett, 2015, 15: 1368-1373

21 Stöber W, Fink A, Bohn E. Controlled growth of monodisperse silica spheres in the micron size range. J Colloid Interface Sci, 1968, 26: $62-69$

Acknowledgements This research was supported by the National Natural Science Foundation of China (51475353, 51475352 and 51375361) and the Tribology Science Fund of the State Key Laboratory of Tribology of China (SKLTKF14A02).

Author contributions Ye X, Cai A and Shao J designed the project and experiments. Ye X, Wu X, Ruan X and Zhang X performed the experiments. Ye $X$ and Zhang $X$ analyzed the results and wrote the manuscript with contribution from all authors

Conflict of interest The authors declare that they have no conflict of interest. 


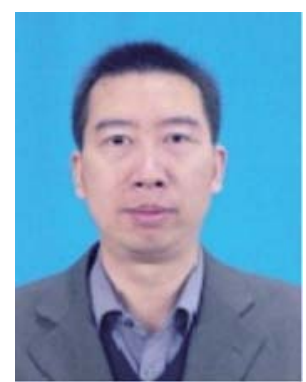

Xiangdong Ye received his PhD degree from the School of Mechanical Engineering, Xi'an Jiaotong University, in 2010. He is currently an associate professor at the School of Mechanical and Electrical Engineering, Xi' an University of Architecture and Technology. His research focuses on techniques for micro/nano-fabrication.

中文摘要 本文提出了一种利用硅橡胶模板对二氧化硅纳米粒子进行图形化组装的方法. 首先, 采用浇筑法制备了硅橡胶基底并通过 激光打标机加工制备了铜掩膜板, 然后对覆盖铜掩膜板后的硅橡胶基底进行金属金的溅射以得到硅橡胶模板. 二氧化硅纳米粒子采用 改进的Stober方法制备, 然后采用离心旋涂方式涂覆到硅橡胶模板表面. 由于硅橡胶模板表面具有图形化的亲水和疏水区域, 而且金表 面的皱化也具有不同的亲水性, 纳米粒子将经历两次去湿过程, 最终围绕金图形化区域形成环状分布. 